\title{
Study of a New Approach about Locating Single-phase-to-ground Fault
}

\author{
Bing Jiang, Chuan Zang, Zhiguo Xiao \\ Jiangsu provincial Key Laboratory of Power \\ Transmission Equipment Technology \\ HoHai University \\ Changzhou, China \\ jiangb@hhuc.edu.cn
}

\author{
Jie Yin \\ East China Power Grid Electric Co. Ltd \\ Suzhou, China \\ 2004yinjie@sina.com
}

\begin{abstract}
First this paper briefly analyzes the electric power transient signal of single phase grounding fault, and then the fault characteristics of zero sequence current and bus voltage is obtained by wavelet transformation. Finally by using genetic algorithm to optimize the original weights of back-propagation neural network, as well as taking the fault characteristics as the input characteristic vector of optimized back propagation neural network, the network is trained and tested. The simulation result shows that the prediction effects and convergence rate of optimized back-propagation network have better performance than traditional back-propagation network. It's relatively error were less than $3 \%$, independent of fault distance, power supply phase angle and the impact of transient resistance.
\end{abstract}

Keywords--single phase grounding fault; wavelet transformation; optimized back-propagation network; genetic algorithm; fault location;

\section{INTRODUCTION}

In power system operation, the probability of single phase grounding fault in small current grounded system is up to $80 \%$. The trend of planning and transforming $10 \mathrm{kV}$ power network in many economically developed regions is that the power is supplied mainly by cable, secondarily overhead line. When single phase grounded fault occurs, grounding residual current is very small. And it does not affect the load power supply. Therefore, the system does not immediately trip. But by now, healthy phase to ground voltage is line voltage in small current grounding system and grounding capacitance current has also increased. As a result, intermittent arc occurs easily at fault point and develops into a permanent grounding fault. It can lead that overvoltage reaches values over the normal phase voltage of 6 to 8 times. Its high amplitude and long duration can bring about a serious threat to existing overvoltage protection. Therefore, the basic guarantee to improve reliability of power network and reduce the accident rate is to determine the single phase grounding fault quickly and accurately.

When single phase grounding fault occurs, it will generate transient fault current and fault voltage. This paper uses wavelet transform to identify transient fault signals. Genetic algorithm has good global search ability. The capacity of non-linear fitting of neural network is well. These studies establish correspondence between features and fault location, and realize the fault location.

\section{CURRENT ANALYSIS OF SINGLE PHASE GROUNDING FAULT}

In isolated neutral system, grounding fault current grounds through imaginary earthing neutral of power transformer that provides single phase grounding fault current with pathway. In Fig. 1, grounding fault current is determined by per-phase capacitance to ground. Assuming that Phase A of Line 2 occurs grounding fault, A phase voltage is zero. Then, for non-fault Line $1, \mathrm{~A}, \mathrm{~B}, \mathrm{C}$ three phase currents of capacitance to ground are:

$$
\begin{aligned}
& \dot{I}_{C_{A 1}}=j \dot{U}^{\prime}{ }_{A} \omega C_{01}, \\
& \dot{I}_{C_{B 1}}=j \dot{U_{B}^{\prime}}{ }_{B} \omega C_{01}, \\
& \dot{I}_{C_{C 1}}=j \dot{U}_{C}^{\prime} \omega C_{01} .
\end{aligned}
$$

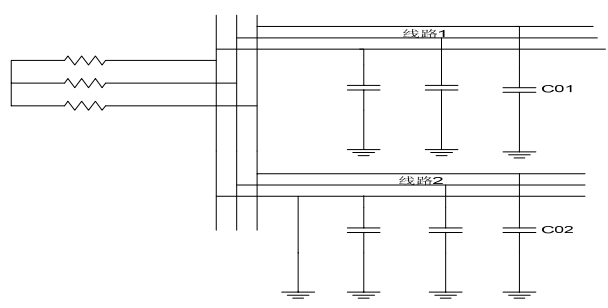

Figure1. Single phase grounding fault in isolated neutral system

Fundamental steady-state zero sequence current in non-fault Line 1 is:

$$
\begin{aligned}
& 3 \dot{I}_{01}=\left(I_{C_{A 1}}+I_{C_{B 1}}+I_{C_{C 1}}\right) \\
& =j 3 \dot{U}_{0} \omega C_{01} .
\end{aligned}
$$

For the fault Line 2, A, B, C, three phase currents of capacitance to ground are:

$$
\begin{gathered}
\dot{I}_{C_{A 2}}=-j 3 \dot{U}_{o} \omega\left(C_{01}+C_{02}\right), \\
\dot{I}_{C_{B 2}}=j \dot{U}_{B}^{\prime} \omega C_{02}, \\
\dot{I}_{C_{C 2}}=j \dot{U}_{C}^{\prime} \omega C_{02} .
\end{gathered}
$$


Zero sequence current of fault Line 2 can be obtained from the above formula:

$$
3 \dot{I}_{02}=\left(\dot{I}_{C_{A 2}}+\dot{I}_{C_{B 2}}+\dot{I}_{C_{c 2}}\right)=-3 \dot{I}_{01} .
$$

Therefore, the zero sequence current of fault line is the sum of all zero sequence currents of non-fault line, and its direction is opposite non-fault line, from line to bus. The current flowing through the fault point is the sum of non-fault phase currents of capacitance to ground. Furthermore, bus voltage will have a change in the fault time. That is to say, zero sequence current and zero sequence voltage of bus, generated by different fault distance, are both different. Therefore, these two variables can be considered as characteristic quantities.

\section{WAVELET TRANSFORM}

Assuming that $\phi(t) \in L^{2}(R) \quad\left(L^{2}(R)\right.$ is square integrable real space, in other words, is signal space of finite energy.), its Fourier transform is $\phi(\omega)$. When $\phi(\omega)$ is satisfied with the condition:

$$
C_{\phi}=\int_{R} \frac{|\phi(\omega)|^{2}}{|\omega|} d \omega<\infty .
$$

$\phi(t)$ is called basic wavelet or mother wavelet.

The convolution type of wavelet transformation of $f(t)$ is:

$$
w_{f}(s, x)=f(x) * \phi_{s}(x)=\frac{1}{s} \int_{R} f(t) \phi\left(\frac{x-t}{s}\right) d t .
$$

In the above formula, if $x$ locates in the neighborhood of $x_{0}$ and $\left|w_{f}\left(s_{0}, x\right)\right| \leq\left|w_{f}\left(s_{0}, x_{0}\right)\right|$, then, $\left(s_{0}, x_{0}\right)$ is modulus maxima of wavelet transform.

If $f(t)$ is the periodic signal, let $f(t)=A f_{0}(t)$.It can be obtained by formula 10 that wavelet transform value of signal is proportional to its amplitude, $W_{f}(s, x) \infty A$. When the power system fault occurs, the amplitude of electrical quantities (voltage, current) go through upheaval (partial phases of electrical quantities also change), and modulus maxima of wavelet transform can be used to determine the signal singularity.

\section{NEURAL NETWORK}

\section{A. Back Propagation Neural Network}

$\mathrm{BP}$ (Back Propagation) neural network is one of the most intensively studied and widely used artificial neural model, its structure shown in Fig.2.

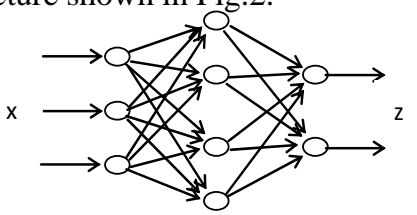

Figure2. The structure of BP neural network
In Fig.2, $\mathrm{x}, \mathrm{z}$ are the network input and output vectors and each neuron is expressed by a node. The network is made up of the nodes of input layer, hidden layer and output layer, and hidden layer may be one layer or a multilayer (in Fig.2, it is a single hidden layer). Nodes from the front layer to the rear layer are linked by the weight coefficients. The learning process of BP neural network is as follows. The input signal transfers from the input layer through the hidden layer to the output layer (forward propagation). If the output layer gets the desired output, the learning algorithm ends; Otherwise, goes backward propagation that through the back-calculation of original join pathway, the error signal (difference between the sample output and internet output) adjusts the weight coefficients of the neurons with gradient descent method to decreases the error signal. The following are specific processes of weight values of each layer (BP learning algorithm):

$$
E=\frac{1}{2}(d-O)^{2}=\frac{1}{2} \sum_{k=1}^{l}\left(d_{k}-o_{k}\right)^{2} .
$$

$E$ is defined as the output errors of the network and is expanded to the hidden layer and the input layer. Under the principle that the errors continue to decrease, adjustment of weight values is proportional to the negative gradient of the errors, namely:

$$
\begin{aligned}
& \Delta w_{j k} \propto-\frac{\partial E}{\partial w_{j k}}, j=0,1,2, \cdots m ; k=1,2, \cdots l ; \\
& \Delta v_{i j} \propto-\frac{\partial E}{\partial v_{i j}}, i=1,2, \cdots n ; j=1,2, \cdots m .
\end{aligned}
$$

By derivation, the adjustment formulas of weight values of each layer can be obtained, written in vector form:

In formulas,

$$
\begin{aligned}
& \Delta W=\eta\left(\delta^{o} Y^{T}\right)^{T}, \\
& \Delta V=\eta\left(\delta^{y} X^{T}\right)^{T} .
\end{aligned}
$$

$X=\left(x_{1}, x_{2}, \cdots, x_{n}\right)^{T}$ is input vector,

$Y=\left(y_{1}, y_{2}, \cdots, y_{m}\right)^{T}$ is output vector of hidden layer,

$O=\left(o_{1}, O_{2}, \cdots, o_{l}\right)^{T}$ is output vector,

$d=\left(d_{1}, d_{2}, \cdots, d_{l}\right)^{T}$ is expected output.

$W=\left[w_{j k}\right]_{m \times l}$ is the weight matrix from hidden layer to the output layer and $V=\left[v_{i j}\right]_{n \times m}$ is from input layer to the hidden layer.

The steps of fault location prediction model of BP neural network are as follows:

(1) Use a three-layer BP network, initialize the network and assign the network parameters and the weight coefficients which should take random numbers.

(2) As shown in TABLE I, the line from $1 \mathrm{~km}$ to $9 \mathrm{~km}$, in increments of $1 \mathrm{~km}$, take three cases that are fault resistance of $50 \mathrm{ohm}$ with phase angle of $0^{\circ}$, fault 
resistance of $500 \mathrm{ohm}$ with phase angle of $0^{\circ}$ and fault resistance of $50 \mathrm{ohm}$ with phase angle of $45^{\circ}$ as horizontal standards. At each point, get zero-sequence current of the fault line and the zero-sequence voltage of bus and the real and imaginary parts of modulus maxima by equation (10)(Morlet complex wavelet) after normalization. Under each standard, 36 data as input training samples, 9 lines distance as output vector, calculate the predicted values for each layer and compares the values with the true values to obtain the error of network output.

(3) Based on error back propagation rule, adjust weight coefficients between hidden layer and input layer, hidden layer and output layer.

(4) Repeat step (2) and (3), until the prediction error satisfying the condition or the training reaching the required number of times.

Based on the above steps, a failure prediction model A can be obtained. Fig. 3 is a training error curve by the standard of the phase of $45^{\circ}$ and fault resistance of $50 \mathrm{ohm}$.

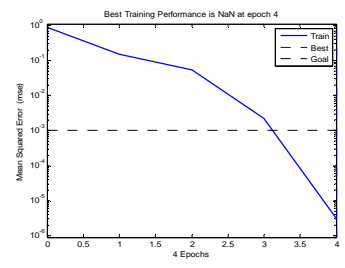

Figure3. Training error curve before optimization

\section{B. Optimized BP neural network}

When the failure prediction model $A$ is established, the weights are random. Practice shows that weights of BP algorithm are random, with low learning efficiency, slow convergence rate, easily failing into local optimum and other shortcomings. Therefore, find other ways to optimize the weights. Here, use genetic algorithm.

In the mid-1960s, based on the research of others, John Holland introduced and established genetic algorithm at University of Michigan. Based on natural selection and genetic theory, genetic algorithm is a global optimization approach. Possible solution of the problem space is seen as the individual that is similar to the chromosome in the group and each individual is encoded into a string form. According to the values calculated by fitness function, simulate biological evolution that contains selection, crossover, mutation and so on to evolve from generation to generation, and ultimately get the optimal solution.

The steps of fault location prediction model of Optimized BP neural network are as follows:

(1) Use a three-layer BP network, initialize population $\mathrm{P}$ including cross scale, crossover probability Pc, mutation probability $\mathrm{Pm}$ and weights between input layer and hidden layer, hidden layer and output layer. In coding, encode with real numbers and initial population.

(2) Calculate and sort the evaluation function of each individual. Select individual network by probability of formula:

$$
P_{s}=\frac{f_{i}}{\sum_{i=1}^{N} f_{i}} .
$$

$f_{i}$ is the match value of $i$, measured by error square sum E, namely:

$$
\begin{gathered}
f(i)=\frac{1}{. E(i)}, \\
E(i)=\sum_{p} \sum_{k}\left(V_{k}-T_{K}\right)^{2} .
\end{gathered}
$$

Where $i=1, \cdots, N$, is the chromosome number, $k$ is the number of nodes in the output layer, $p$ is the number of learning sample and $T_{k}$ is the teacher signal.

(3) For individuals $G_{i}$ and $G_{i+1}$ with probability $p_{c}$, take crossover operator to generate new individuals $G_{i}^{\prime}$ and $G_{i+1}^{\prime}$, and the individuals without crossover operator replicate directly.

(4) For $G_{j}$ with probability Pm, take mutation operation to generate new individual $G_{j}^{\prime}$.

(5) Insert the new individuals into the population $P$ and calculate their evaluation function.

(6) If find the satisfactory individuals, then end. Otherwise, go to step (3).After achieving the required performance, decode the best individuals in the group to get the connection weights of optimized network [8]. Then, follow four steps of model A, and a new fault localization prediction model $\mathrm{B}$ will be obtained.

After building the model $\mathrm{B}$, that the training error curve under the standard of the phase of $45^{\circ}$ and fault resistance of $50 \mathrm{ohm}$ can be archived, as shown in Fig. 4

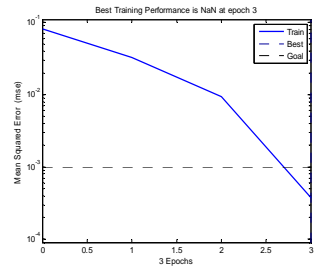

Figure4. The optimized training error curve

Contrasting Fig. 3 and Fig.4, it can be concluded that to achieve the same accuracy, such as , before optimization require 3.2 times, 2.6 times just after optimization. Therefore, genetic algorithm is applied to train the learning of neural network weights. The trained neural network converges faster, and complex network better reflects the superiority of genetic algorithm.

\section{SimULATIONS AND ANALYSIS}

In this paper, construct single phase grounding fault model of $10 \mathrm{kV}$ isolated neutral system with Simulink in MATLAB, shown in Fig.5, and predict the location by fault localization prediction model $\mathrm{B}$. 


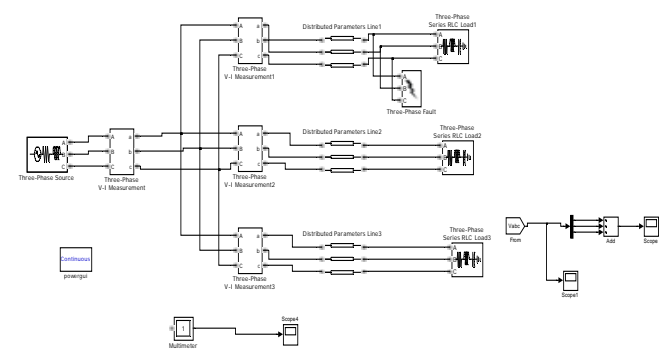

Figure5. Simulation model of $10 \mathrm{kV}$ single phase grounding fault

The lengths of three lines are respective $20 \mathrm{~km}, 100 \mathrm{~km}$, $100 \mathrm{~km}$. Take the first line for experiment, which the line is from $1 \mathrm{~km}$ to $9 \mathrm{~km}$, in increments of $1 \mathrm{~km}$. Take three cases that are fault resistance of $50 \mathrm{ohm}$ with phase angle of $0^{\circ}$, fault resistance of $500 \mathrm{ohm}$ with phase angle of $0^{\circ}$ and fault resistance of $50 \mathrm{ohm}$ with phase angle of $45^{\circ}$ as horizontal standards. These three criteria are standard 1 and standard 2 , Standard 3. At each point, get zero-sequence current of the fault line and the zero-sequence voltage of bus, convert them into .mat file, put the file into workspace of MATLAB and get the real and imaginary parts of modulus maxima by Morlet complex wavelet. Normalize these data as training samples and put them into neural network that is optimized by genetic algorithm. After training the network, use testing samples for testing and get the test results of the fault line by anti-normalization to calculate the percentage of relative error. The results are shown in TABLE I.

TABLE I Testing results and relative errors under various standards

\begin{tabular}{|c|c|c|c|c|c|c|}
\hline $\begin{array}{l}\text { Fault } \\
\text { Line } \\
\text { dista } \\
\text { nce }\end{array}$ & $\begin{array}{l}\text { Test } \\
\text { distanc } \\
\text { e of } \\
\text { standar } \\
\text { d1 }\end{array}$ & $\begin{array}{l}\text { Relat } \\
\text { ive } \\
\text { error } \\
\text { (\%) }\end{array}$ & $\begin{array}{l}\text { Test } \\
\text { distanc } \\
\text { e of } \\
\text { standar } \\
\text { d2 }\end{array}$ & $\begin{array}{l}\text { Relat } \\
\text { ive } \\
\text { error } \\
\text { (\%) }\end{array}$ & $\begin{array}{l}\text { Test } \\
\text { distanc } \\
\text { e of } \\
\text { standar } \\
\text { d2 }\end{array}$ & $\begin{array}{l}\text { Relat } \\
\text { ive } \\
\text { error } \\
(\%)\end{array}$ \\
\hline 1 & $4^{1.021}$ & 2.14 & $7^{1.011}$ & 1.17 & $7^{1.001}$ & 0.17 \\
\hline 2 & $3^{2.009}$ & 0.47 & $5^{2.006}$ & 0.33 & $4^{1.948}$ & 2.58 \\
\hline 3 & $4^{2.995}$ & 0.15 & $3^{3.002}$ & 0.08 & $1^{2.998}$ & 0.06 \\
\hline 4 & $6^{3.994}$ & 0.14 & $8^{3.994}$ & 0.13 & $4^{4.001}$ & 0.04 \\
\hline 5 & $3^{4.989}$ & 0.21 & $0^{4.996}$ & 0.08 & $9^{5.026}$ & 0.54 \\
\hline 6 & $9^{5.984}$ & 0.25 & $9^{5.985}$ & 0.24 & $0^{5.988}$ & 0.03 \\
\hline 7 & $3^{6.993}$ & 0.10 & $8^{7.000}$ & 0.11 & $7^{7.020}$ & 0.30 \\
\hline 8 & $0^{7.989}$ & 0.13 & $1^{8.000}$ & 0.01 & $7^{7.941}$ & 0.73 \\
\hline 9 & $0^{8.980}$ & 0.22 & $7^{9.000}$ & 0.08 & $6^{8.870}$ & 1.44 \\
\hline
\end{tabular}

TABLE I illustrates that wavelet transform and optimized neural network are good for fault location. Under three standards, relative errors of the testing distances are less than three percent. And it can be drawn by the horizontal standards that this locating method is basically not affected by fault position, fault resistance and phase angle.

At the same time, the testing distances of fault line with A model are obtained. The compare results are shown in Fig.6, the horizontal axis is the fault line distance and the vertical axis is the output error (the difference between testing distance and fault line distance).It can be drawn that the output error of optimized BP neural network is closer to zero, that is, the testing results is closer to the sample values.

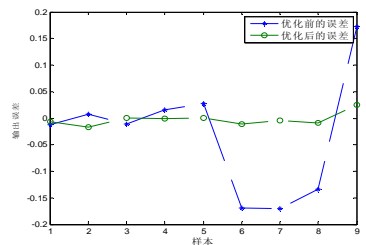

Figure6. Errors in the testing results before and after optimization

\section{CONCLUSION}

Wavelet transform as a modern signal processing, very suitable for the analysis of power system transient process, combined with the improved BP network input and output good nonlinear mapping ability, to complete the classification and location of the fault. Combined with wavelet transform, improved BP network which has good nonlinear mapping capabilities of input and output, is able to complete the fault classification and location. Testing results shows that this locating method is basically not affected by fault position, fault resistance and phase angle. In addition, it avoids to trap into a local minimum, accelerates the speed of network processing and provides new impetus for intelligent diagnosis. Meanwhile, genetic algorithm can optimize the learning rules and structure of neural network, worthy of further study.

\section{REFERENCES}

[1] Chaari O, Meunier M, Brouaye F. Wavelets: A New Tool For the Resonant Grounded Power Distribution Systems Relaying[J]. IEEE Transactions on Power Delivery, 1996, 11(3): 1301-1308.

[2] Yao X. A Review Of Evolutionary Artificial Neural Networks[J]. International Journal of Intelligent Systems, 1993, 8(4):539-567.

[3] Mallat S, Hwang W L. Singularity Detection and Processing with Wavelets[J]. IEEE Trans on Inform Theory, 1992, 38(2): 617-643.

[4] Pan H. Application of BP neural network based on genetic algorithm[J]. Computer Application, 2005, 25(12): 2777-2779.

[5] Dong T Z, Guo J H, Lv J. Research of Fault Detection in Transmission Line based on Neural Network[J]. Journal of System Simulation, 2009, 21(15): 4903-4907.

[6] Cui X M, Sun C X, Li X. Analysis of Real Wavelet and Complex Wavelet Transform in Extracting PD Signal Feature[J]. Transactions of China Electrotechnical Society, 2004, 19(7): 90-94.

[7] Lv Y Z. Power System Faults Detection Based on Singularity Detection of Wavelet[J]. Journal of Jilin University (Information Science Edition), 2009, 27(2): 156-161.

[8] Li X J, Kou Y S. Interharmonic Detection Algorithm Based on Amplitude and Phase Information of Morlet Complex Wavelet 
Transform[J]. Computer engineering \& Software, 2010, 31(10): 49-53.

[9] Li J K. the Research of Single-Phase-Ground Line Selection and Fault Location based on Wavelet Packet Neural Network in Distribution System[D]. Nanning: Guangxi University, 2009.
[10] Zhu K, Wang Z L. Proficient in MATLAB Neural Network[M].Beijing: Publishing House of Electronics Industry, 2009: 317-325.

[11] Gao M J, Zhao Y, Tan A L. Study on Genetic Wavelet Neural Network based Multi-sensor Information Fusion Technique[J].Chinese Journal of Scientific Instrument, 2007, 28(11): 2103-2107. 